n.tronos

glyndwhr

Glyndŵr University

Glyndŵr University Research Online

Computing

Computer Science

9-1-2004

\title{
A Hybrid Computational Electromagnetics Formulation for Simulation of Antennas Coupled to Lossy and Dielectric Volumes
}

\author{
R A. Abd-Alhameed \\ Peter S. Excell \\ Glyndwr University, p.excell@glyndwr.ac.uk \\ M A. Mangoud
}

Follow this and additional works at: http://epubs.glyndwr.ac.uk/cair

Part of the Computer Engineering Commons, and the Signal Processing Commons

\section{Recommended Citation}

Abd-Alhameed, R.A., Excell, P.S., \& Mangoud, M.A. (2004) 'A hybrid computational electromagnetics formulation for Simulation of antennas coupled to lossy and dielectric volumes.'IEEE Transactions on Broadcasting, 50(3), 253-259

This Article is brought to you for free and open access by the Computer Science at Glyndŵr University Research Online. It has been accepted for inclusion in Computing by an authorized administrator of Glyndŵr University Research Online. For more information, please contact

d.jepson@glyndwr.ac.uk. 


\title{
A Hybrid Computational Electromagnetics Formulation for Simulation of Antennas Coupled to Lossy and Dielectric Volumes
}

\begin{abstract}
A heterogeneous hybrid computational electromagnetics method is presented, which enables different parts of an antenna simulation problem to be treated by different methods, thus enabling the most appropriate method to be used for each part. The method uses a standard frequency-domain moment-method program and a finite-difference time-domain program to compute the fields in two regions. The two regions are interfaced by surfaces on which effective sources are defined by application of the Equivalence Principle. An extension to this permits conduction currents to cross the boundary between the different computational domains. Several validation cases are examined and the results compared with available data. The method is particularly suitable for simulation of the behavior of an antenna that is partially buried, or closely coupled with lossy dielectric volumes such as soil, building structures or the human body.
\end{abstract}

Keywords

hybrid computational electromagnetics formulation, antennas, lossy, dielectric volumes, frequency domain, equivalence principle, antenna simulation

\section{Disciplines}

Computer Engineering | Electrical and Computer Engineering | Signal Processing

\section{Comments}

This paper was published in IEEE Transactions on Broadcasting in 2004. The definitive version is available at www./ieeexplore.ieee.org (C2004 IEEE. Personal use of this material is permitted. However, permission to reprint/republish this material for advertising or promotional purposes or for creating new collective works for resale or redistribution to servers or lists, or to reuse any copyrighted component of this work in other works must be obtained from the IEEE. This material is presented to ensure timely dissemination of scholarly and technical work. Copyright and all rights therein are retained by authors or by other copyright holders. All persons copying this information are expected to adhere to the terms and constraints invoked by each author's copyright. In most cases, these works may not be reposted without the explicit permission of the copyright holder. 


\title{
A Hybrid Computational Electromagnetics Formulation for Simulation of Antennas Coupled to Lossy and Dielectric Volumes
}

\author{
Raed A. Abd-Alhameed, Peter S. Excell, and Mohab A. Mangoud
}

\begin{abstract}
A heterogeneous hybrid computational electromagnetics method is presented, which enables different parts of an antenna simulation problem to be treated by different methods, thus enabling the most appropriate method to be used for each part. The method uses a standard frequency-domain moment-method program and a finite-difference time-domain program to compute the fields in two regions. The two regions are interfaced by surfaces on which effective sources are defined by application of the Equivalence Principle. An extension to this permits conduction currents to cross the boundary between the different computational domains. Several validation cases are examined and the results compared with available data. The method is particularly suitable for simulation of the behavior of an antenna that is partially buried, or closely coupled with lossy dielectric volumes such as soil, building structures or the human body.
\end{abstract}

Index Terms-Antennas, computational electromagnetics, finite difference time domain (FDTD), ground plane antennas, hybrid methods, lossy dielectrics, method of moments (MoM).

\section{INTRODUCTION}

$\mathbf{E}$ LECTROMAGNETIC field computation algorithms are widely used for the prediction of the behavior of antennas of all types. The algorithms broadly fall into two categories, integral equation methods and differential equation methods, which have significantly different strengths and weaknesses. Integral equation methods (often known as the method of moments) are widely used for simulation of broadcasting antennas since they can simulate the behavior of arbitrarily shaped metallic structures with relative ease and with a good level of fidelity. However, problems arise if the antenna is associated with substantial dielectric volumes (especially the important cases of lossy dielectrics such as earth, sea water and the human body). The problem arises because integral equation methods inherently compute the coupling between each discretization element in the model and every other element: this does not cause a problem for metal because it is considered to be effectively impenetrable and hence only the surface has to be discretized; further, wires and thin rods can be represented as one-dimensional elements. Penetrable dielectric, on the other

Manuscript received September 1, 2000; revised May 3, 2004.

R. A. Abd-Alhameed is with the Department of Electronics and Telecommunications, School of Engineering, Design and Technology, University of Bradford, Bradford, BD7 1DP U.K.

P. S. Excell is with the Department of Electronic Imaging and Media Communications, University of Bradford, Bradford BD7 1DP, U.K.

M. A. Mangoud is with the Electronics and Communications Engineering Department, Arab Academy for Science and Technology, Alexandria, Egypt.

Digital Object Identifier 10.1109/TBC.2004.834008 hand, has to be represented with a three-dimensional assembly of volumetric elements. Leaving aside the mathematical complexity of modeling such elements, the key difficulty that arises is that, in being a three-dimensional structure, the number of elements required in the model rapidly becomes very large and the computational task can become unmanageable.

An alternative approach for penetrable dielectrics that is computationally much more tractable, is to use a differential equation method, such as the finite difference time-domain method (FDTD). This is a well known and highly developed algorithm that directly implements a discretized form of Maxwell's Equations. It is very efficient and relatively easy to implement, but it has one major disadvantage in that it requires the problem space to be discretized into three-dimensional rectangular elements. This results in a significant deficiency in the modeling of structures with surfaces that are not parallel to the principal axes, since the surfaces then have a stepped shape (known as 'staircasing') as a result of conformance to the rectangular quantization grid. This poses particular problems when attempts are made to model linear (wire-type) antennas with components that are not parallel to the axes.

These two methods have unique advantages in their own domain of application but it is not possible to apply either to the other domain without significant disadvantages (e.g., using integral equation methods to model the ground or using FDTD to model wires). A method was thus developed to enable both of them to be used for a single problem, combining them via a hybridization algorithm in which a bounding surface was established on which fictitious equivalent sources were considered to exist.

The first stage of hybridization between these methods was achieved by analyzing each part of a problem with the most appropriate method [1], [2], the coupling between these methods being computed by using the Equivalence Principle theorem. In this theorem the objects should not be physically connected, but separated by a distance of at least a moderate fraction of a wavelength to place the equivalence-principle surface in isolation between them [3].

If the objects are physically connected and have some conductivity, a different treatment is required to allow the conduction current to cross the boundary between the two methods, applying a special treatment to ensure current continuity across the surface. Earlier work [3]-[6] is here extended for the case where the source region is subdivided, one part replaced by equivalent surface currents using the equivalence principle (computed by MoM) and the other part handled with the use of 


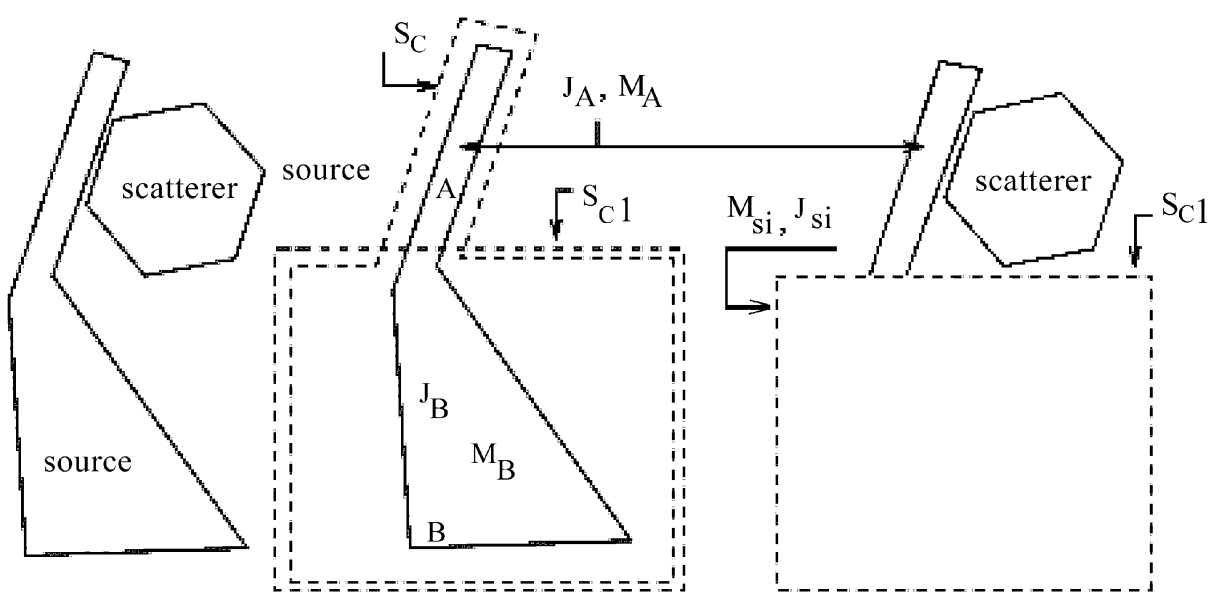

(a)

(b)

(c)

Fig. 1. The basic geometry of the problem, with differing approaches to partition.

direct impressed currents (suitable to couple with FDTD). An industry-standard frequency-domain MoM program [7] was used, to aid acceptance in practical applications, although it can easily be modified for use with any MoM program.

Coupling between the scatterer and source regions is calculated using the Reaction Theorem, with appropriate modifications to the MoM program to take account of the fact that the basis and weighting functions are not the same.

\section{Summary OF THE METHOD}

Fig. 1(a) shows two regions, one enclosing the source, the other enclosing the scatterer. The source region is further subdivided into two sub-regions, A and B, as shown in Fig. 1(b). In general there is no physical attachment between source region $\mathrm{A}$ and the scatterer region, but the scatterer can be very close to, or attached to, the source region B if it has a dielectric surface. The source region was modeled using MoM whereas the scatterer was modeled using FDTD. In Fig. 1(b) the surface $\mathrm{S}_{\mathrm{c}}$ encloses the entire source region. The fields due to the induced currents from both source sub-regions A and B over the closed surface $\mathrm{S}_{\mathrm{c} 1}$ can be computed in order to evaluate the surface currents $\mathrm{J}_{\mathrm{si}}$ and $\mathrm{M}_{\mathrm{si}}$ :

$$
\begin{aligned}
\mathbf{M}_{\mathbf{s i}} & =\left(\mathbf{E}\left(\mathbf{J}_{\mathbf{A}}, \mathbf{M}_{\mathbf{A}}\right)+\mathbf{E}\left(\mathbf{J}_{\mathbf{B}}, \mathbf{M}_{\mathbf{B}}\right)\right) \times \hat{\mathbf{n}} \\
\mathbf{J}_{\mathbf{s i}} & =\hat{\mathbf{n}} \times\left(\mathbf{H}\left(\mathbf{J}_{\mathbf{A}}, \mathbf{M}_{\mathbf{A}}\right)+\mathbf{H}\left(\mathbf{J}_{\mathbf{B}}, \mathbf{M}_{\mathbf{B}}\right)\right)
\end{aligned}
$$

where $\mathrm{J}_{\mathrm{A}}, \mathrm{J}_{\mathrm{B}}, \mathrm{M}_{\mathrm{A}}$, and $\mathrm{M}_{\mathrm{B}}$ are the electric and magnetic currents of source regions $\mathrm{A}$ and $\mathrm{B}$; $\hat{\mathrm{n}}$ is the unit vector normal to the surface $S_{c 1}$. Now let the part of the surface $S_{c}$ adjacent to sub-region $\mathrm{A}$ be coincident with the surface of the conducting source structure within A. The currents on $\mathrm{S}_{c}$ then become the surface currents on the conductor, which can be transferred to an FDTD model by treating them as impressed currents. The whole source region is then modeled using the FDTD method with impressed currents replacing sub-region A and a surface of equivalent currents replacing sub-region B (see Fig. 1(c)). The hard (impressed) sources for a perfectly conducting surface $\left(\mathrm{M}_{\mathrm{A}}=0\right)$ considered in region $\mathrm{A}$ can be given in FDTD as follows:

$$
\mathbf{E}=\mathbf{E}_{\mathrm{FDTD}}+\mathbf{J}_{\mathbf{A}} /(\varepsilon / \Delta \mathrm{t}+\sigma / 2)
$$

where $\mathbf{E}_{\mathrm{FDTD}}$ represents the normal electric field finite-difference time-domain updating equations with a total/scattered field formulation [2], $\Delta \mathrm{t}$ is the time step, and $\varepsilon$ and $\sigma$ are the permittivity and the conductivity of the surrounding medium, respectively. For region $B$, that is surrounded by the closed surface $S_{c 1}$, the difference equations at the surface for the FDTD method can be stated as follows:

$$
\begin{aligned}
\mathbf{E} & =\mathbf{E}_{\mathrm{FDTD}}+\left.\mathbf{J}_{\mathrm{si}}\right|_{S_{c 1}} /(\varepsilon / \Delta \mathrm{t}+\sigma / 2) \\
\mathbf{H} & =\mathbf{H}_{\mathrm{FDTD}}-\left.\mathbf{M}_{\mathrm{si}}\right|_{\mathrm{S}_{c 1}} \Delta \mathrm{t} / \mu
\end{aligned}
$$

where $\mathrm{J}_{\mathrm{si}}$ and $\mathrm{M}_{\mathrm{si}}$ are the surface electric and magnetic currents, and with the suffix $\mathrm{S}_{\mathrm{c} 1}$ are only those defined at the surface $\mathrm{S}_{\mathrm{c} 1} \cdot \mu$ is the permeability of the medium.

The voltage induced on the source region A due to the backscattered fields from the scatterer can be given as:

$$
\mathrm{V}_{\mathrm{A}}=\left(\mathrm{E}_{\mathrm{free}}-\mathrm{E}_{\mathrm{scat}}\right) \cdot \Delta \mathrm{s}
$$

where $E_{\text {free }}, E_{\text {scat }}$ are the induced electric fields on the region A without and with the scatterer, and $\Delta \mathrm{s}$ is the FDTD cell size. The voltage induced in region B (the scattered field region) is found by using MoM, employing the reaction theorem:

$$
\begin{aligned}
& \mathrm{V}_{\mathrm{B}}=\int_{S_{B}} \mathrm{~J}_{\mathrm{ts}} \cdot \mathrm{E}_{i b} d s_{B} \\
& \mathrm{~V}_{\mathrm{B}}=\int_{S_{c 1}^{-}}\left(\mathrm{E}_{\mathrm{ts}} \cdot \mathrm{J}_{i b}-\mathrm{H}_{\mathrm{ts}} \cdot \mathrm{M}_{i b}\right) d s_{c 1}^{-}
\end{aligned}
$$

where:

$$
\begin{aligned}
\mathrm{E}_{\mathrm{ib}} & =-j \omega \mathrm{A}(\mathrm{r})-\nabla \mathrm{V}(\mathrm{r})-\frac{1}{\varepsilon} \nabla \times \mathrm{F}(\mathrm{r}), \\
\mathbf{A}(\mathbf{r}) & =\mu \int_{\mathrm{S}_{c 1}^{-}} \mathbf{J}_{i b} g\left(\mathbf{r}, \mathbf{r}^{\prime}\right) d s_{c 1}^{-}, \\
V(\mathbf{r}) & =\frac{-j}{\omega \varepsilon} \int_{\mathrm{S}_{\mathrm{c} 1}^{-}} \nabla_{s}^{\prime} \cdot \mathbf{J}_{i b} g\left(\mathbf{r}, \mathbf{r}^{\prime}\right) d s_{c 1}^{-}, \\
\mathbf{F}(\mathbf{r}) & =\varepsilon \int_{\mathrm{S}_{c 1}^{-}} \mathbf{M}_{i b} g\left(\mathbf{r}, \mathbf{r}^{\prime}\right) d s_{c 1}^{-},
\end{aligned}
$$




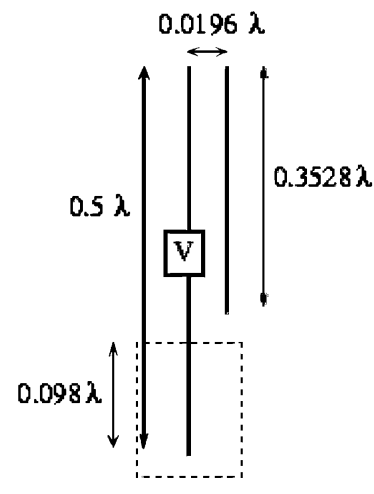

(a)

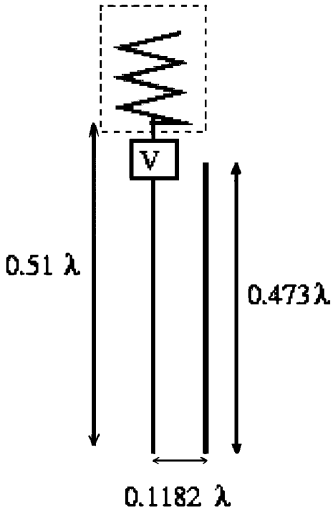

(b)
Fig. 2. The geometry of the examples 2 and 3 .

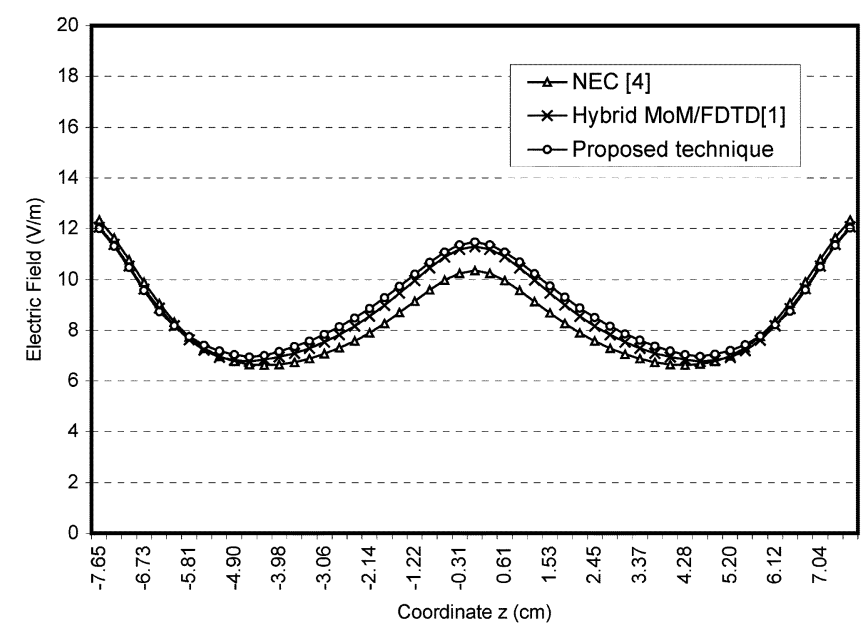

Fig. 3. Comparison between the computed near electric fields of a dipole in free space using three different methods (Example1).

and

$$
g\left(\mathbf{r}, \mathbf{r}^{\prime}\right)=\frac{e^{-j k\left|\mathbf{r}-\mathbf{r}^{\prime}\right|}}{4 \pi\left|\mathbf{r}-\mathbf{r}^{\prime}\right|}
$$

is the free space Green's function.

The vectors $\mathbf{r}$ and $\mathbf{r}^{\prime}$ apply to the source and observation points respectively. $\mathrm{S}_{\mathrm{B}}$ is the conducting surface area of the structure within region $\mathrm{B} . \mathbf{J}_{\mathrm{ts}}$ is the electric test function used on the wire or the patch. It is usually a uniform pulse to account for the excitation voltage at the centre of the wire segment and the patch for the MoM, if using NEC-2 [7]. $\mathbf{E}_{\mathrm{ts}}$ and $\mathbf{H}_{\mathrm{ts}}$ are the electric and magnetic fields respectively for the test function $\mathbf{J}_{\mathrm{ts}}$. It should be noted that, in applying (7), there is no magnetic test function specified in NEC-2 [7]. Since $\mathbf{E}_{\mathrm{ts}}$ and $\mathbf{H}_{\mathrm{ts}}$ can be obtained easily using NEC, after ignoring the sinusoidal basis functions, and assuming that the cell meshing used in FDTD is very small compared to the operating wavelength, (8) can be reduced by ignoring the surface integral and evaluating the voltage back-scattered corresponding to the centre of the cell surface, as shown below:

$$
V_{b}=\sum_{n_{S_{c 1}^{-}}}\left(\mathbf{E}_{\mathrm{ts}}\left(\mathbf{r}, \mathbf{r}^{\prime}\right) \cdot \mathbf{J}_{i b n}-\mathbf{H}_{\mathrm{ts}}\left(\mathbf{r}, \mathbf{r}^{\prime}\right) \cdot \mathbf{M}_{i b n}\right) a_{n}
$$

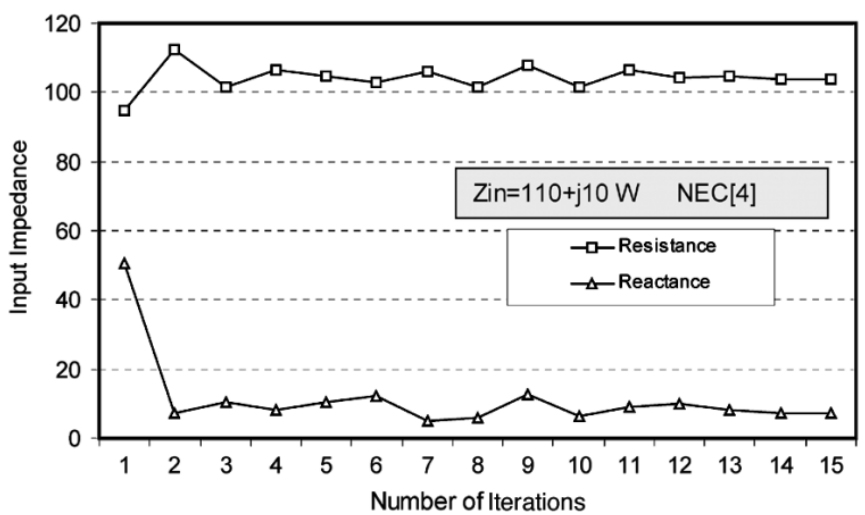

Fig. 4. Input Impedance of the Source Dipole with scatterer of Example 2 versus number of iterations.

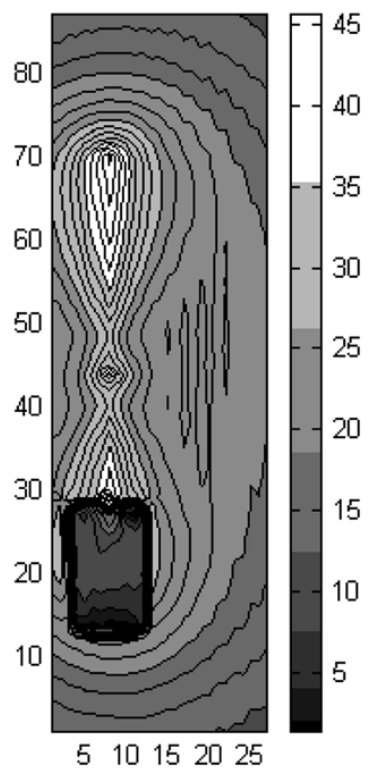

(a)

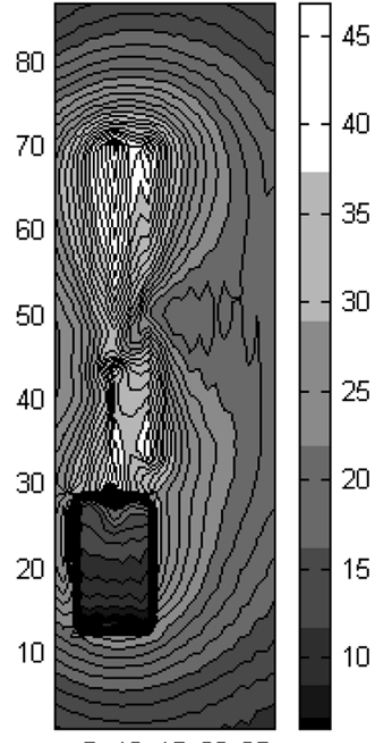

510152025

(b)
Fig. 5. Near field contour at central y-z plane for Example 2: (a) without the scatterer; (b) with the scatterer.

where $\mathbf{r}_{\mathrm{n}}^{\prime}$ is the position vector of the centre of the cell surface and $a_{\mathrm{n}}$ is the surface area of the cell. Therefore $\mathbf{J}_{\mathrm{ibn}}$ and $\mathbf{M}_{\mathbf{i b n}}$ are considered to be the equivalent surface currents at the centre of the surface cell $\mathrm{n}$. At the boundary of the hard source and the equivalent surface $\left(S_{c 1}\right)$ FDTD requires at least 2 to 3 cells of additional margin to recalculate the voltage induced in region $B$ as seen from Fig. 1(b). When the voltages have been obtained, the new currents can be computed and the method can be repeated until the steady state is reached.

\section{SimUlations AND RESULTS}

Near fields and input impedance are taken to be convenient but rigorous validation parameters for the following examples:

1) A perfectly electrically conducting (PEC) half-wavelength dipole with radius $0.001 \lambda$, in free space, was first considered. The equivalent surface treatment was arbitrarily chosen to enclose $70.4 \%(0.352 \lambda)$ of the length of the dipole, whereas the rest of the dipole was replaced 


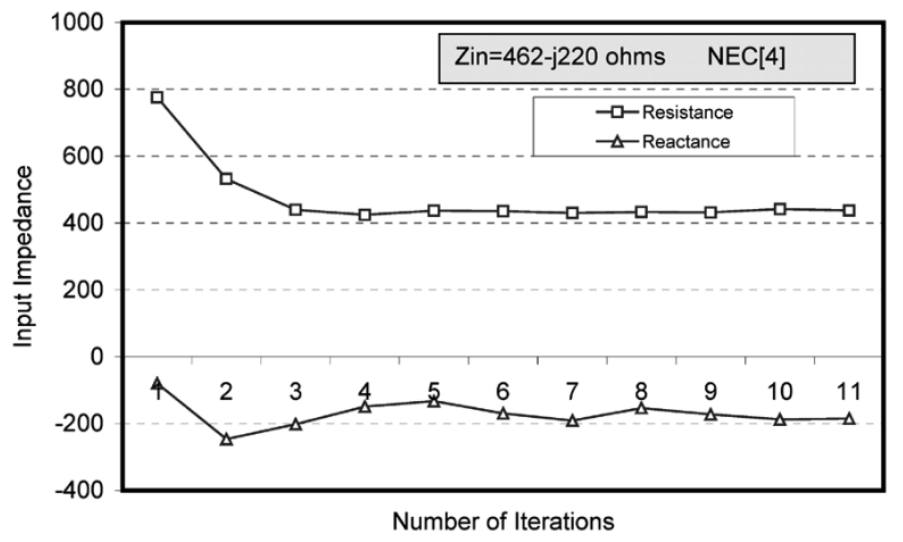

Fig. 6. Input impedance of the semi-helical dipole with scatterer of Example 3 , versus number of iterations (for hybrid treatment).

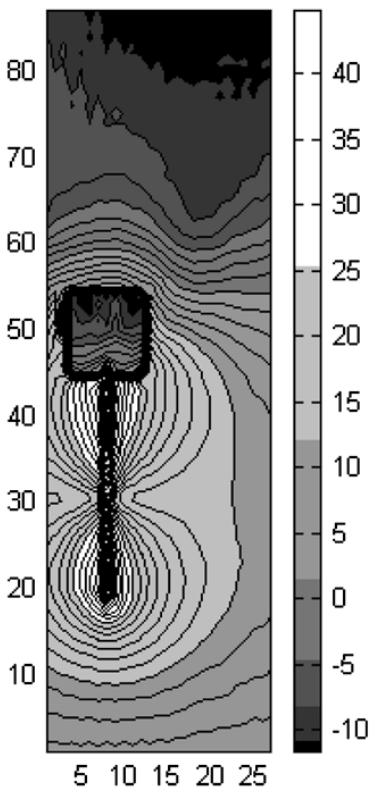

(a)

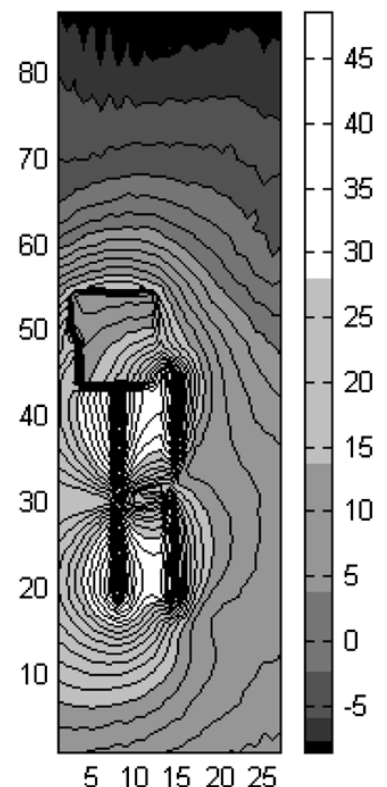

(b)
Fig. 7. Near field contours at central y-z plane for Example 3: (a) without the scatterer; (b) with the scatterer.

by the impressed currents. The free space electric field on a line parallel to the dipole at a transverse distance of $0.0346 \lambda$ was examined, as shown in Fig. 3. The results are in good agreement with those from hybrid [5] and MoM programs [7].

2) A scatterer was introduced, in the form of a PEC rod of length $0.3528 \lambda$ and radius $0.001 \lambda$, parallel to the source dipole at a distance $0.0196 \lambda$ (see Fig. 2(a)). The scatterer is located in the region of the impressed current source. The input impedance of the source dipole versus the number of iterations is shown in Fig. 4. The results are in good agreement with those from a uniform MoM treatment [7]. The electric field contours in the $y-z$ plane, which contains the source and the scatterer, are shown in Fig. 5 for the cases with and without the scatterer. This figure clarifies the methodology used and the technique of the current crossing the boundary (i.e., the equivalent surface principle).

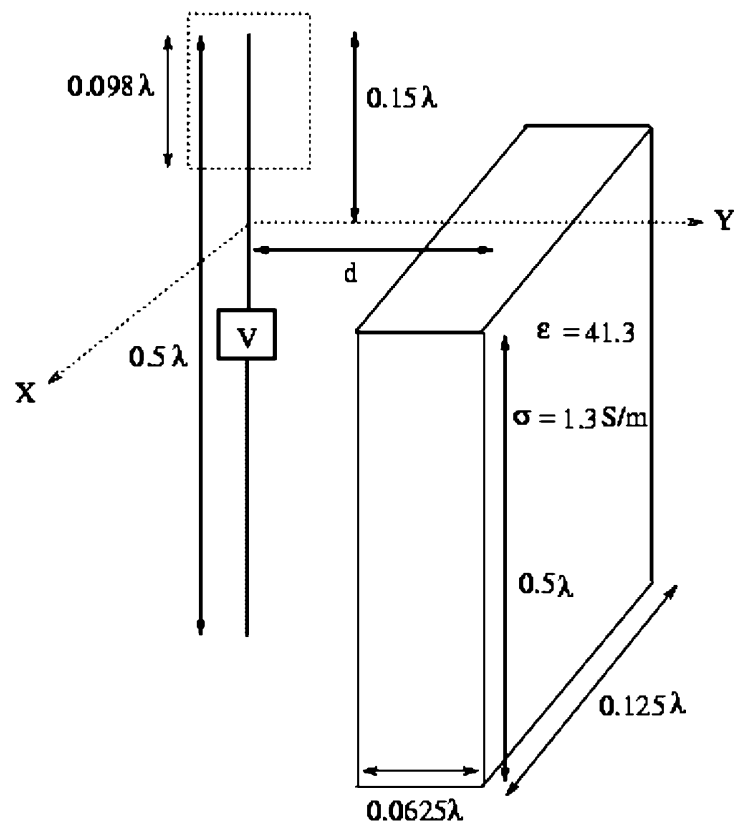

Fig. 8. The geometry problem for Example 4.

3) In this example, illustrated in Fig. 2(b), the source region contains a dipole with total conductor length $1.0 \lambda$ and radius $0.001 \lambda$. One of dipole arms is coiled into a helix with three turns of radius $0.12 \lambda$ and pitch $0.004 \lambda$. This simulates a canonical form of a transmitter cabinet directly feeding a helical antenna adjacent to a scatterer, in a case where the cabinet is intended to be simulated by FDTD. The scatterer is a wire of length $0.473 \lambda$ and radius $0.001 \lambda$. The scatterer and the source are separated by $0.01182 \lambda$. The equivalent surface encloses the helix region while the impressed current treatment is used for the other arm of the dipole. The input impedance versus the number of iterations is shown in Fig. 6. The results are also in good agreement with a nonhybrid treatment [7]. Similarly to Fig. 5, the electric field contour for this example is presented in Fig. 7. The plane displayed contains the scatterer and the second arm of the source dipole that is represented by the straight wire. The near field distribution shows the important advantage of the method over the conventional hybrid technique [4], [5], [8]-[10] that requires a more substantial separation distance between the source and the scatterer.

4) In this case the scatterer region is taken to be a slab of lossy dielectric. The sheet dimensions are $0.125 \lambda \times$ $0.0625 \lambda \times 0.5 \lambda$ and it was modeled with a relative permittivity of 41.3 and conductivity of $1.3 \mathrm{Sm}^{-1}$, as presented in Fig. 8: these material properties would correspond to a highly hydrated and conducting material such as biological tissue, but seawater is not greatly dissimilar and the same principle applies to any other penetrable lossy dielectric, such as earth or construction materials. The input impedance versus the number of iterations required to reach steady state solution, for three different distances, is shown in Fig. 9. The results are in excellent 


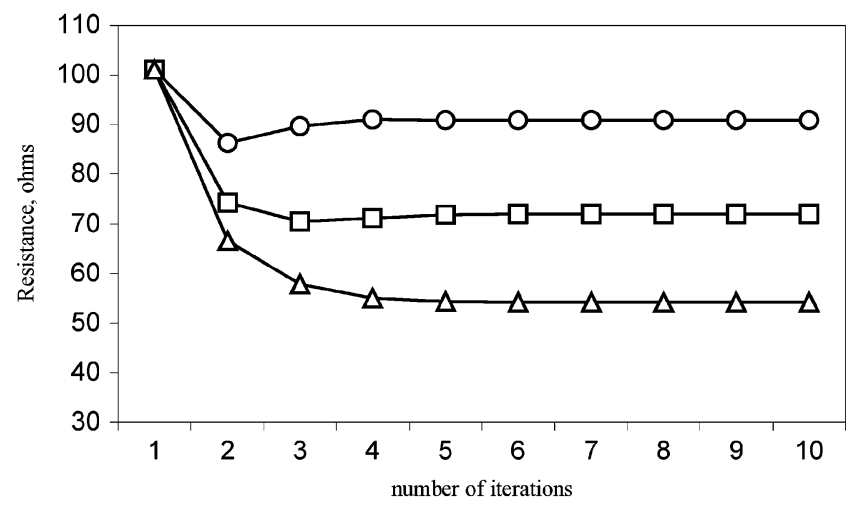

-0.2 wavelength $-\square-0.15$ wavelength $\triangle-0.1$ wavelength

(9a)

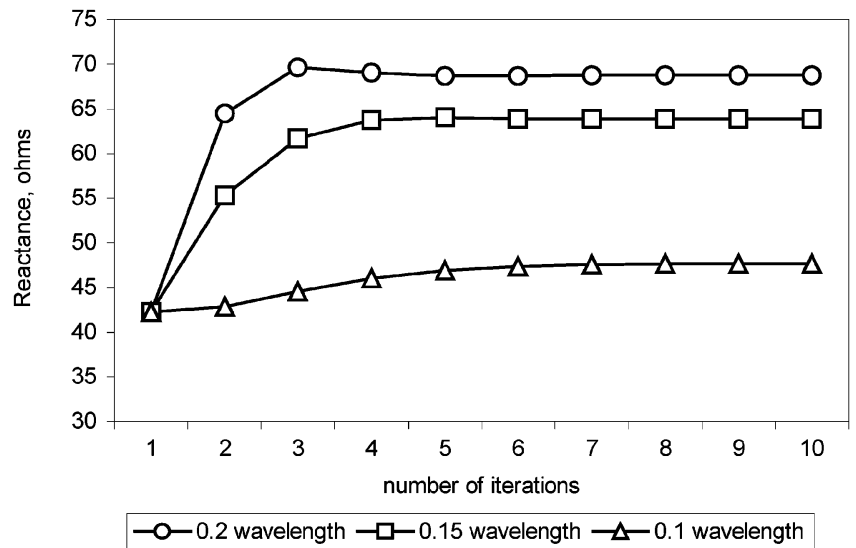

(9b)

Fig. 9. The input resistance and reactance of the source dipole in Example 4, for three different distances from the dielectric.

agreement with the data in [11]. The far field pattern for two different planes is shown in Fig. 10.

5) A typical HF broad-band duo-conical monopole antenna over an infinite conducting perfect ground plane is modeled as an example of a broadcasting antenna and to insure the ability of the method to account for the ground plane effect in the presence of the current-crossing boundary. The antenna, shown in Fig. 11, has the following properties: $34.3 \mathrm{~m}$ high, $45.0 \mathrm{~m}$ across, $110^{\circ}$ apex angle and $2.2^{\circ} \mathrm{sag}$ angle. The antenna is chosen to be simulated as operating within the short wave band at $3 \mathrm{MHz}$, with a VSWR of 1.75 at the input port (the input port is placed in the segment of the antenna attaching it to the ground plane). The antenna and ground models inside the FDTD problem space are shown in Fig. 12. The problem space is set to be $72 \times 72 \times 67$ cells and the equivalence-principle surface is $50 \times 50 \times 40$ cells. The antenna currents outside this surface are represented by the impressed currents as described in the summary of the method. The near field magnitude of the dominant vertically-polarized electric field component, observed at $27 \mathrm{~m}$ radial distance from the origin, as a function of height (i.e., distance in the z-direction) is shown in Fig. 13. The results are in excellent agreement with those
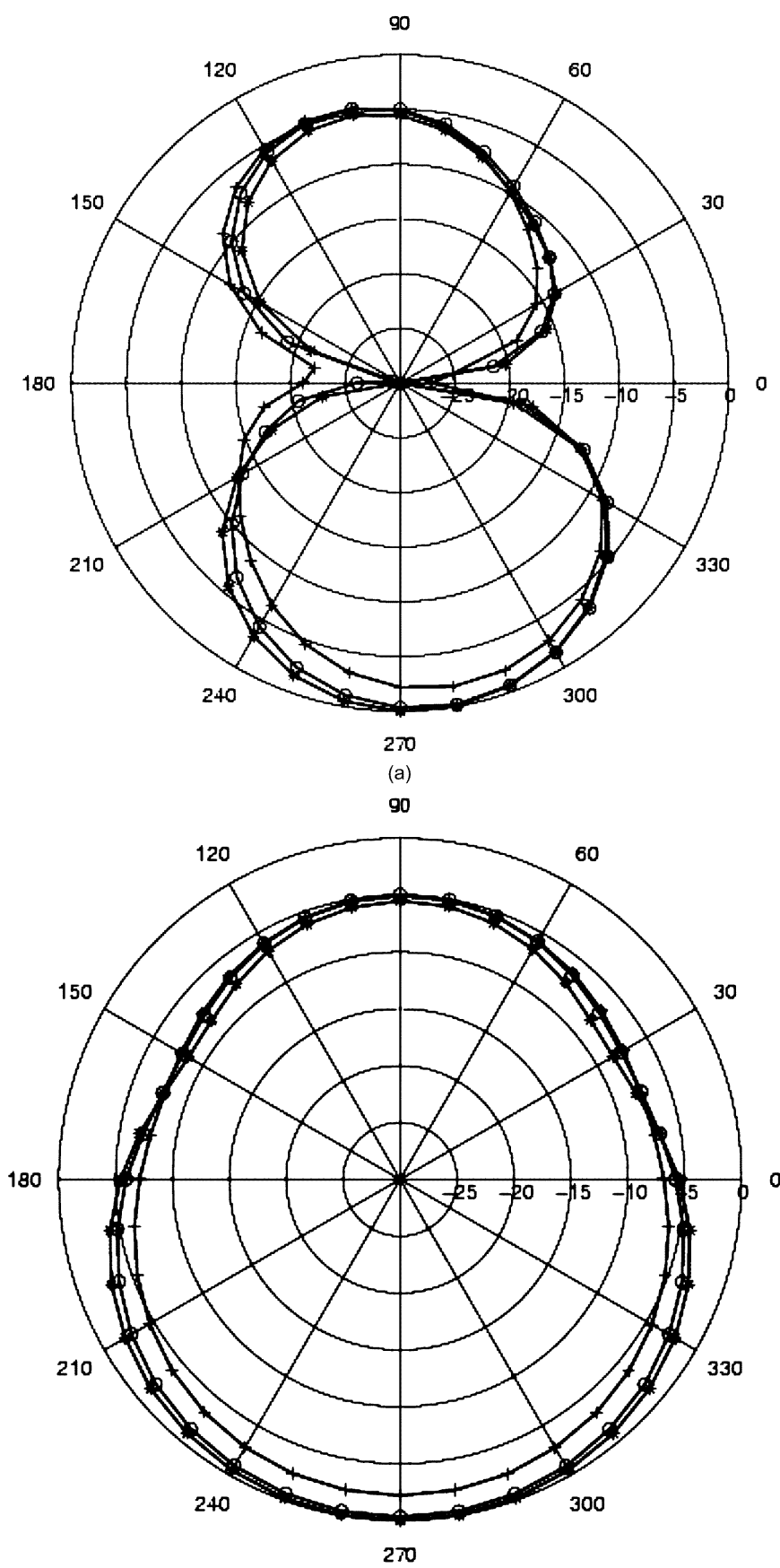

(b)

Fig. 10. The far-field $\mathrm{E}_{\theta}$ component for Example 4, at two different cuts: (a) vertical cut at $\phi=90^{\circ}$; (b) horizontal cut at $\theta=90^{\circ}$, for three different separation distances from the dielectric. $(-+-0.1 \lambda,-0-0.15 \lambda,-*$ $-0.2 \lambda)$.

from NEC-2 [7]. The hybrid method, however, has much greater flexibility than pure MoM, such as NEC-2, to handle differing types of ground and arbitrarily-shaped volumes of lossy dielectric.

\section{CONCLUSION}

A novel computational method has been described which enables the simulation of broadcasting antennas with a much-enhanced combination of verisimilitude and accuracy than was 


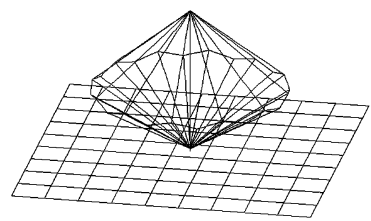

(a)

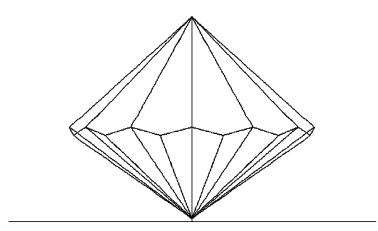

(b)

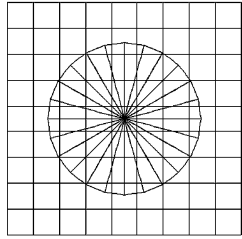

(c)

Fig. 11. Different views of the duo-conical monopole antenna on a perfect ground plane (a) 3D (b) front view (c) top view.

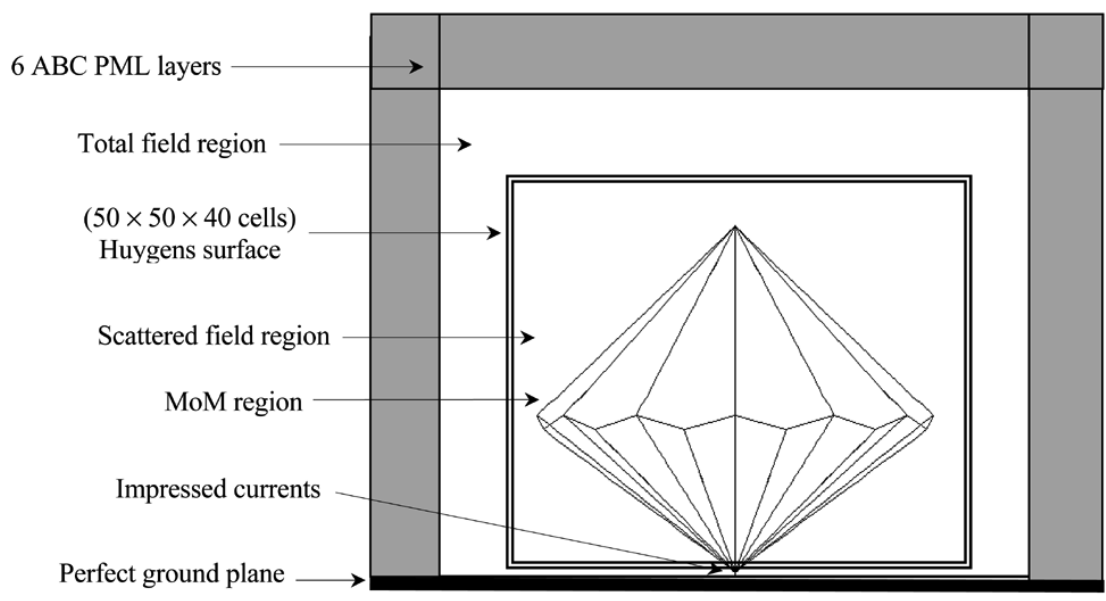

(a)

(b)

Fig. 12. FDTD problem space details of Example 5.

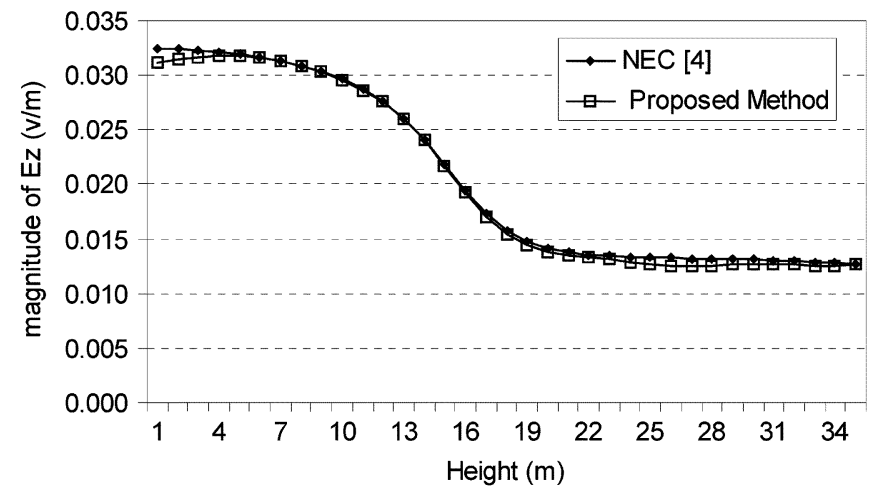

Fig. 13. Dominant (vertically polarized) electric field magnitude $\left(E_{z}\right)$ versus height at $27 \mathrm{~m}$ radial distance from the origin.

previously possible. This is because it combines the very different advantages of integral equation (method of moments) and differential equation (finite difference time-domain method) computational algorithms which are, individually, well-adapted to the simulation of metallic and penetrable dielectric structures, respectively. By combining the strengths of these two methods in a hybrid formulation an optimum system is obtained that is particularly powerful for the simulation of antennas that are close to, or partially buried in, lossy ground and the method is equally applicable to the related problems of antennas in or near sea water and those adjacent to the human body. Basic hybrid methods do not permit current flow across the boundary separating the two domains, but the treatment presented here has demonstrated a method to achieve this: this is particularly useful for antennas driven against a ground plane.
Good convergent results are obtained after a few iterations. The results are in good agreement with the available data.

\section{ACKNOWLEDGMENT}

The author would like to thank Mr. J. A. Vaul, Bradford University, for valuable discussions and for his help with FDTD matters.

\section{REFERENCES}

[1] E. K. Miller, L. Medgyesi-Mitschang, and E. H. Newman, Eds., Computational Electromagnetics: Frequency-Domain Method of Moments. New York, NY: IEEE Press, 1992.

[2] A. Taflove and S. C. Hagness, Computational Electrodynamics: The Finite-Difference Time-Domain Method, 2nd ed. Boston, MA: Artech House, 2000.

[3] R. A. Abd-Alhameed, P. S. Excell, J. A. Vaul, and M. A. Mangoud, "Hybrid treatment for electromagnetic field computation in multiple regions," Electron. Lett., vol. 34, pp. 1925-1926, 1998.

[4] G. Cerri, P. Russo, A. Schiavoni, G. Tribellini, and P. Bielli, "MoM-FDTD hybrid technique for analysing scattering problems," Electron. Lett., vol. 34, pp. 433-440, 1998.

[5] R. A. Abd-Alhameed, P. S. Excell, J. A. Vaul, and M. A. Mangoud, "Computation of radiated and scattered fields using separate frequencydomain moment-method regions and frequency-domain MoM-FDTD hybrid methods," in IEE Conf. Antennas \& Propagation, York, 1999, pp. 53-57.

[6] M. A. Mangoud, R. A. Abd-Alhameed, and P. S. Excell, "Simulation of human interaction with mobile telephones using hybrid techniques over coupled domains," IEEE Trans. Microwave Theory and Techniques, vol. 48, pp. 2014-2021, Nov. 2000.

[7] G. H. Burke and A. J. Poggio, "Numerical Electromagnetics Code (NEC): Method of Moments," US Naval Ocean Systems Center, San Diego, CA, Rep. no. TD116, 1981.

[8] R. A. Bretones, R. Mittra, and G. Martin, "Hybrid NEC/FDTD approach for analysing electrically short thin-wire antennas located in proximity of inhomogeneous scatterers," Electron. Lett., vol. 35, pp. 1594-1596, 1999. 
[9] N. Rogier, F. Olyslager, and D. De Zutter, "A new hybrid FDTD-BIE approach to model electromagnetic scattering problems," IEEE Microwave and Guided Wave Lett., vol. 3, pp. 138-140, 1998.

[10] A. Monorchio and R. Mittra, "A hybrid finite-element-difference time-domain (FE/FDTD) technique for solving complex electromagnetic problems," IEEE Microwave and Guided Wave Lett., vol. 8, pp. 93-95, 1998.

[11] R. A. Abd-Alhameed and P. S. Excell, "Analysis of dielectrically-loaded wire, strip and patch antennas, using method of moments," in Proc. IEE Conf. Computation in Electromagnetics, 1996, pp. 306-311.

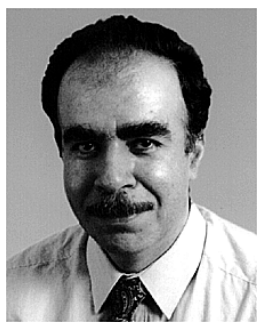

Raed A. Abd-Alhameed was born in Basrah, Iraq in 1959. He received the B.Sc. and M.Sc. degrees from Basrah university, Iraq, in 1982 and 1985 respectively, and the Ph.D. degree from the University of Bradford, UK, in 1997, all in Electrical Engineering. From 1997 to 1999 he was a Postdoctoral Research Fellow at the Uuniversity of Bradford, specializing in computational modeling of electromagnetic field problems, microwave nonlinear circuit simulation, signal processing of pre-adaption filters in adaptive antenna arrays and simulation of active inductance. In 2000 he was appointed as a lecturer in Electronic Engineering at the University of Bradford. In August 2003 he was promoted to Senior Lecturer in Applied Electromagnetics at the same University. His current research interests include hybrid electromagnetic computational techniques, antenna design, low SAR antennas for mobile handsets, RF mixers and active antennas. Dr. Abd-Alhameed is a member of the Institution of Electrical Engineers and the Institute for Learning and Teaching in Higher Education.

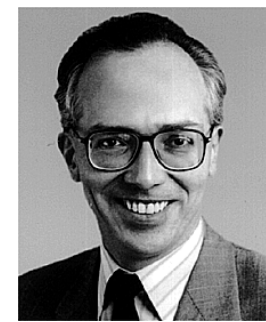

Peter S. Excell is Professor of Applied Electromagnetics and Director of Research in the School of Informatics at the University of Bradford, UK, where he has worked since 1971. He obtained his B.Sc. in Engineering Science from the University of Reading in 1970 and his Ph.D. from the University of Bradford in 1980 for research in electromagnetic hazards. His research interests cover computational electromagnetics, EMC, antenna design and bioelectromagnetics and he has published over 200 papers; he also holds two patents. He is a Senior Member of the Institute of Electronics and Electrical Engineers, a Fellow of the Institution of Electrical Engineers and a Chartered Engineer.

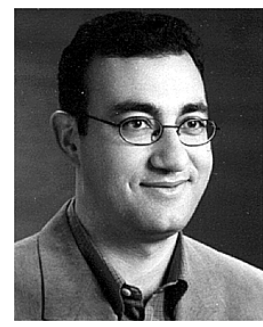

Mohab A. Mangoud was born in Alexandria, Egypt, in 1971. He received the B.Sc. and M.Sc. degrees, both in Electrical Engineering, from Alexandria University, Egypt, in 1993 and 1996 respectively, and the Ph.D. degree in Electronic and Electrical Engineering from the University of Bradford, UK in 2001. He is currently an Assistant Professor in the Electronics and Communications Engineering Department, Arab Academy for Science and Technology and Maritime Transport (AAST), Alexandria, Egypt, where he has worked since 1993. He is responsible for teaching antennas and wireless communications courses. His research interests include modeling of electromagnetic systems, Antenna Design and smart antennas. Dr. Mangoud is a member of the Institute of Electrical and Electronics Engineers. 